\title{
Electromagnetic characteristics of filaments for 3D printing with carbon fillers in the microwave range
}

\author{
Grigoriy E. Kuleshov ${ }^{1, *}$, Alexander V. Badin ${ }^{1}$, Kirill V. Bilinsky ${ }^{1}$, and Kirill V. Dorozhkin ${ }^{1}$ \\ ${ }^{1}$ National Research Tomsk State University, 634050, Tomsk, Russia
}

\begin{abstract}
The results of a study of the complex permittivity and electromagnetic response from polymer composite materials obtained by additive technology from $3 \mathrm{D}$ printing filaments containing various carbon fillers are presented. New radio filaments for 3D printing with MWCNTs have been created. Investigated PLA-Conductive plastics may be used to create a shielding coating or narrowband absorbers for microwave range.
\end{abstract}

\section{Introduction}

Over the past decades, components and devices obtained by additive technologies have begun to be actively applied and implemented [1-4]. Additive technologies have a number of advantages compared to standard ones: fewer operations, waste-free use of initial components, ease of development and optimization of products in CAD engineering programs, and high technology [5-7]. Recently, the manufacture of composite materials based on plastics for 3D printing with the addition of fillers effectively interacting with electromagnetic radiation is developing more and more actively [8-10]. Over the past 5 years, the number of works devoted to the development and research of such radiomaterials has grown more than ten times. The use of additive technology with radiofilaments allows you to create completely new elements and compositional structures, and also makes it possible to significantly speed up and reduce the cost of the production of protective coatings and radio components for microwave devices. This theme is extremely important and has great prospects for practical use.

Among filaments for 3D printing, conductive plastics stand out. As a rule, they contain various carbon structures (carbon fibers, nanotubes, graphene) in their composition [11-13]. For their use as radio materials, it is necessary to know the exact information about the values of their complex permittivity and conductivity.

This paper presents the results of a study of microwave electromagnetic characteristics of industrially produced and self-made filaments for 3D printing containing various carbon fillers.

\section{Objects of research}

The objects of study are composite radio materials manufactured using additive technology (3D printing) from industrially produced and self-made plastic filaments containing various

* Corresponding author: grigorij-kge@sibmail.com 
carbon fillers. As filaments for 3D printing were used: 1) Proto-pasta PLA-Conductive "ProtoPlant Inc." (USA), with conductive carbon: resistivity $10^{2}-10^{3} \mathrm{Ohm} / \mathrm{cm} ; 2$ ) ABSConductive filament "Mellow" (China) containing carbon fibers: resistivity $10^{4}-10^{5}$ $\mathrm{Ohm} / \mathrm{cm}$; 3) Self made filaments: ABS + MWCNT with an average diameter of $9.4 \mathrm{~nm}$ in concentrations up to $5 \mathrm{wt} . \%$.

When making the radiofilament independently, the following scheme was used (Fig. 1). At the first stage, ABS plastic was crushed and dissolved in acetone, and then MWCNTs were added. Next, the resulting mixture was thoroughly mixed and subjected to ultrasonic dispersion for 10 minutes at a power of 75 watts. After complete polymerization, the resulting material was crushed and hot extruded to create a filament. At the final stage, using the obtained radiofilament, a sample in the form of a washer with a thickness of $h=2.25 \mathrm{~mm}$ with an inner diameter of $d=3 \mathrm{~mm}$ and an external $D=7 \mathrm{~mm}$ were printed on a $3 \mathrm{D}$ printer.

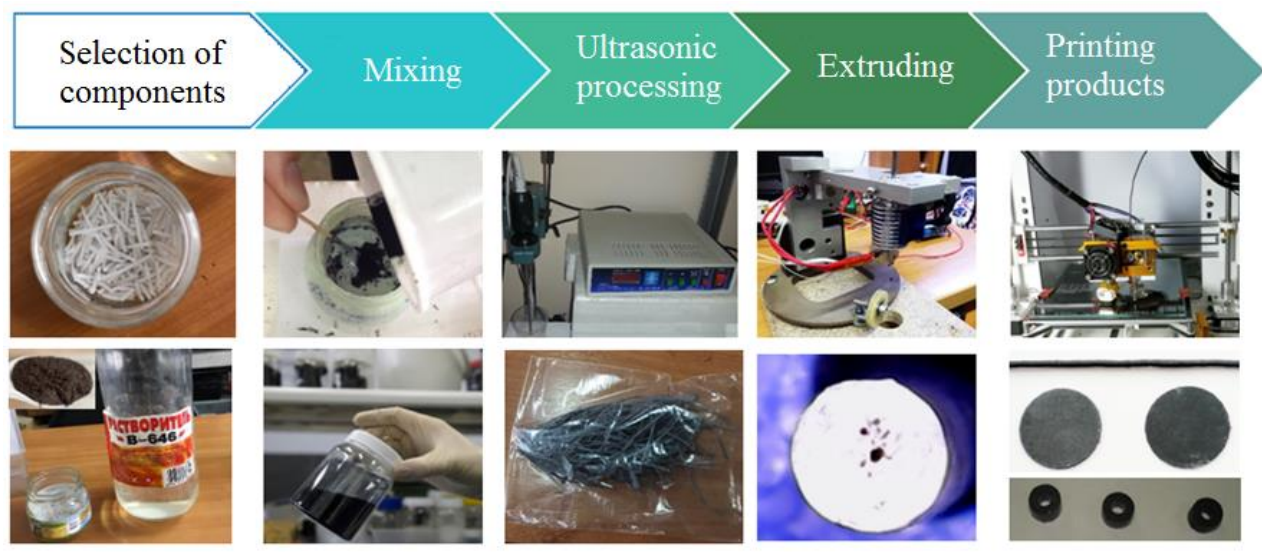

Fig. 1. The process of manufacturing samples based on ABS plastic and MWCNTs.

\section{Measurement technique and experimental results}

The electromagnetic characteristics of the composites were studied using the waveguide method in a coaxial measuring cell on the vector circuits analyzer P4M-18 manufactured by "Mikran" in the frequency range from 0.1 to $18.00 \mathrm{GHz}$. In this case, to the "to pass" measurement schemes were used (Fig. 2). It allows you to measure S-parameters and their phase. Based on the obtained data by the Becker-Jarvis method [14], spectra of complex permittivity of the material were calculated.

Then, from the obtained data on the complex permittivity, with the virtual absence of magnetic properties, we simulated the electromagnetic response from a layer of material located in free space (transmission coefficient) and on the metal (reflection coefficient). For the respective coefficients, distribution plots depending on the thickness and frequency of the layer were obtained.

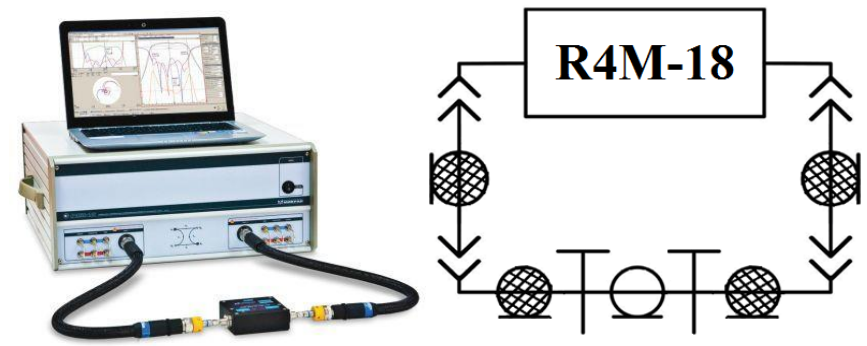

Fig. 2. Appearance and block diagram of the experimental setup. 


\section{Results and discussion}

As a result of measurements and calculations, the spectra of the complex permittivity of composites based on carbon-containing filaments for 3D printing were obtained. The frequency dependences of the real part of the complex permittivity are shown in Fig. 3.

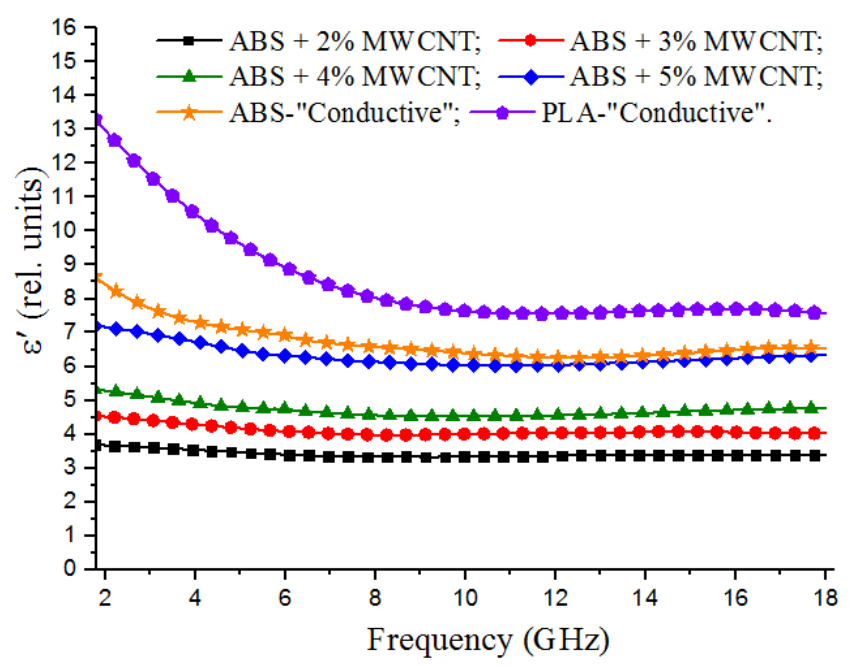

Fig. 3. Frequency dependence of the real part of the complex permittivity for a composite samples based on carbon-containing filaments.

The graphs show that largest values of the real part of the complex dielectric constant have samples of materials based on PLA-Condactive plastic (13-8 rel. units) and ABSCondactive plastic (8.5-7.5 rel. units). Moreover, at lower frequencies they have higher values of $\varepsilon$ ', which decrease with increasing frequency, due to the presence of conductive properties in the materials. Samples based on ABS plastic with the addition of MWCNTs take $\varepsilon^{\prime}$ values within 3.5-7 rel. units. With an increase in the concentration of MWCNTs, $\varepsilon^{\prime}$ also increases. For these samples, with increasing frequency $\varepsilon^{\prime}$ practically does not change.

Next, Fig. 4 shows the frequency dependences of the imaginary part of the complex permittivity.

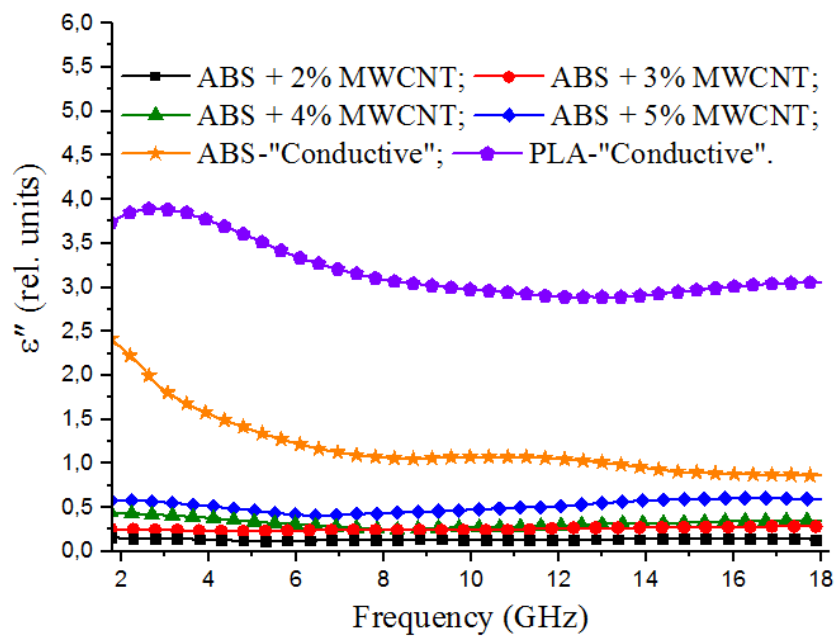

Fig. 4. Frequency dependence of the imaginary part of the complex permittivity for a composite based on carbon-containing filaments. 
It follows from the graphs that the largest values of the imaginary part of the complex permittivity have samples of materials based on PLA-Condactive plastic (3.8-3 rel. units) and ABS-Condactive plastic (2.3-1 rel. units). And samples based on ABS plastic with the addition of MWCNTs take $\varepsilon^{\prime \prime}$ values not exceeding 0.75 rel. units. With an increase in the concentration of MWCNTs, $\varepsilon$ " also increases. It should be noted that with an increase in the frequency $\varepsilon^{\prime \prime}$ in conductive plastics decreases. This is due to the contribution of losses to the conductivity in the imaginary part of the complex permittivity.

The following are the results of modeling the electromagnetic response. For modeling, as an example, the sample with the highest values of dielectric constant was selected. Namely, coatings based on PLA-Condactive plastic placed in free space and coatings deposited on metal.

In free space, the shielding ability of the material was calculated, i.e. spectra of transmission coefficient depending on the thickness of the coating (Fig.5).

The graph shows that in the frequency range $2-18 \mathrm{GHz}$, with increasing frequency and thickness, the shielding properties of the coatings from plastic PLA-Conductive increase. This material has good shielding ability. With a layer thickness of more than $3 \mathrm{~mm}$ at frequencies of more than $6 \mathrm{GHz}$, less than $25 \%$ of microwave radiation penetrates through the composite. Moreover, more than $50 \%$ of the radiation is absorbed, and the rest is reflected. With increasing thickness and frequency, more and more radiation is shielded. At the maximum considered indicators, less than $6 \%$ of the radiation passes.

For the coating deposited on the metal, the reflectance spectra were calculated depending on the thickness of the coating (Fig. 6).

As can be seen from the graph, in the range from 5 to $18 \mathrm{GHz}$, a composite based on PLAConductive has large reflection losses, but at a certain thickness. It can be used as an extremely efficient narrow-band absorber at the considered frequencies. So at a frequency of 5-6 GHz with a thickness of 4-5 mm and at a frequency of $17-18 \mathrm{GHz}$ with a thickness of 2 $\mathrm{mm}$, the attenuation of reflected radiation can reach $-35 \mathrm{~dB}$ (more than 3,000 times in power).

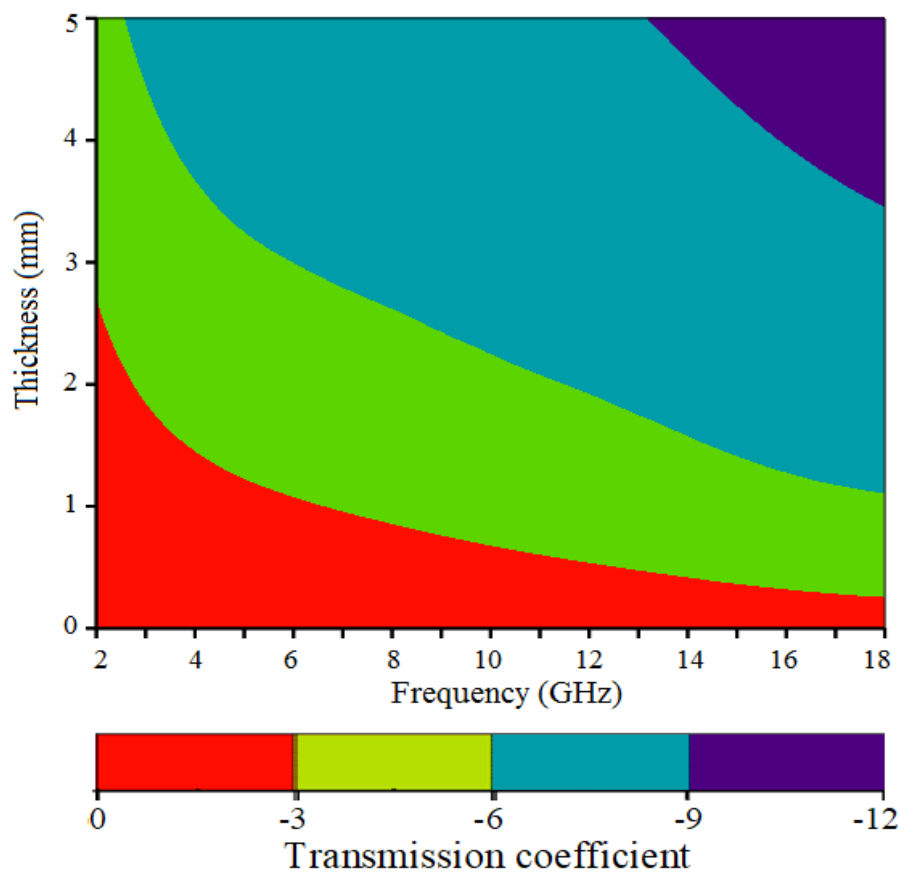

Fig. 5. Distribution of transmission coefficient by frequency and layer thickness for a material based on PLA-Conductive located in free space. 


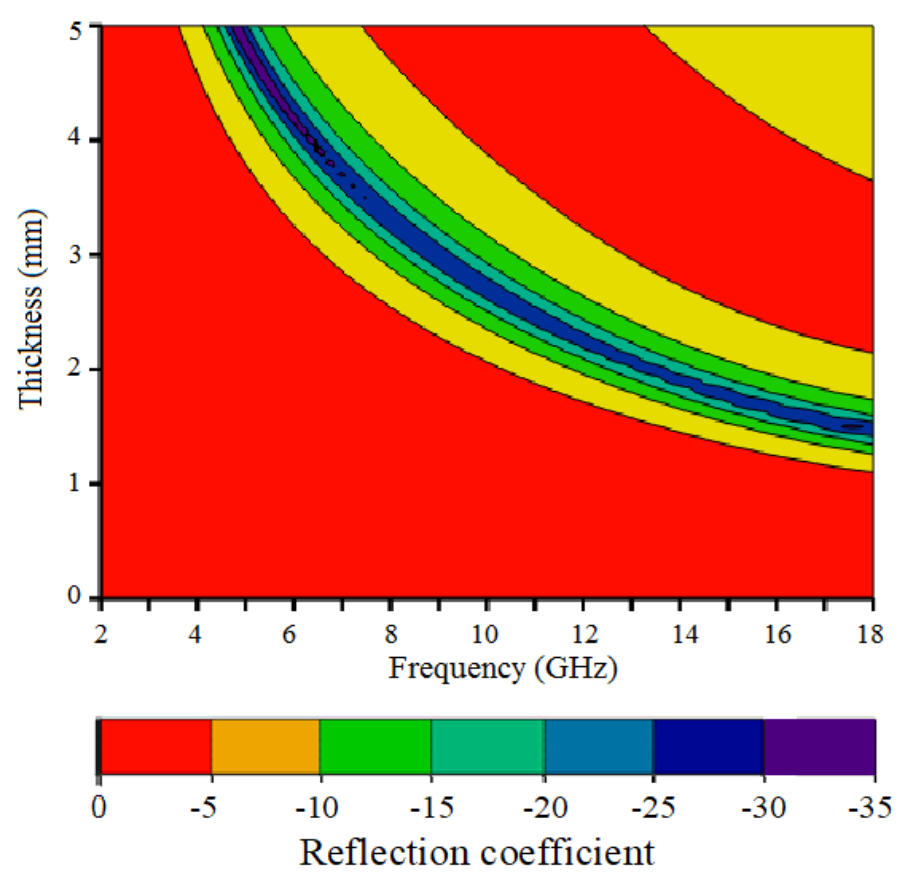

Fig. 6. Distribution of reflection coefficient by frequency and layer thickness for a material based on PLA-Conductive located on a metal.

The research was supported by The Tomsk State University competitiveness improvement programme.

\section{References}

1. S. Koziel, J. Meng, J. W. Bandler, J. W. Bakr, Q. S. Cheng, IEEE Trans. on MW. Theor. and Tech., 57, 383 (2009)

2. V. Laur, M. Kaissar Abboud, A. Maalouf, D. Palessonga, A. Chevalier, J. Ville, APMC, 1318 (2019)

3. P. Pa, Z. Larimore, P. Parsons, M. Mirotznik, Elect. Lett., 51, 1561 (2015)

4. S. Johann, F. William, P. Aurélien, T. Olivier, D. Nicolas, S. Bila, V. Serge, P. JeanBaptiste, R. Gramond, EuMC 2016, 373 (2016)

5. E. Drahi, A. Gupta, S. Blayac, S. Saunier, P. Benaben, Phys. St. Sol. (A) App. and Mat. Sci., 211, 1301 (2014)

6. M. D'Auria, W. J. Otter, J. Hazell, B. T. W. Gillatt, C. Long-Collins, N. M. Ridler, S. Lucyszyn, IEEE Trans. on Comp., Pack. and Manuf. Tech., 5, 1339 (2015)

7. J. G. Hester, S. Kim, J. Bito, T. Le, J. Kimionis, D. Revier, C. Saintsing, W. Su, B. Tehrani, A. Traille, B.S. Cook, M. M. Tentzeris, Proc. of the IEEE, 103, 583 (2015)

8. Y. Wang, F. Castles, P. S. Grant, Mat. Res. Soc. Sym. Proc, 1788, 29 (2015)

9. M. RaviPrakash, S. Anuj, K. Balasubramanian, Mat. Lett., 238, 214 (2019)

10. S. Mishra, P. Katti, S. Kumar, S. Bose, Chem. Eng. J., 357, 384 (2019)

11. A. V. Badin, G. E. Kuleshov, K. V. Dorozhkin, G. E. Dunaevskii, V. I. Suslyaev, V. A. Zhuravlev, IRMMW-THz, 8509938 (2018) 
12. Z. Hu, F. Chen, J. Xu, Q. Nian, D. Lin, C. Chen, M. Zhang, J. of Al. and Comp., 746, 269 (2018)

13. M. Hasan, J. Saa'diAbdul, A. H. Mohammed, A. H. Yazan, W. Salah, Phys.B: Cond. Matt., 418, 41 (2013)

14. K. Chalapat, K. Sarvala, J. Li, G.S. Paraoanu, IEEE Trans. on MW. Theor. and Tech., 57, 2257 (2009) 